\title{
LETTER
}

\section{Genetic variability in a population of arbuscular mycorrhizal fungi causes variation in plant growth}

\author{
Alexander M. Koch, Daniel Croll \\ and Ian R. Sanders* \\ Department of Ecology \\ and Evolution, University of \\ Lausanne, Lausanne, \\ Switzerland \\ *Correspondence: E-mail: \\ ian.sanders@unil.ch
}

\begin{abstract}
Different species of arbuscular mycorrhizal fungi (AMF) alter plant growth and affect plant coexistence and diversity. Effects of within-AMF species or within-population variation on plant growth have received less attention. High genetic variation exists within AMF populations. However, it is unknown whether genetic variation contributes to differences in plant growth. In our study, a population of AMF was cultivated under identical conditions for several generations prior to the experiments thus avoiding environmental maternal effects. We show that genetically different Glomus intraradices isolates from one AMF population significantly alter plant growth in an axenic system and in greenhouse experiments. Isolates increased or reduced plant growth meaning that plants potentially receive benefits or are subject to costs by forming associations with different individuals in the AMF population. This shows that genetic variability in AMF populations could affect host-plant fitness and should be considered in future research to understand these important soil organisms.
\end{abstract}

\section{Keywords}

Arbuscular mycorrhizal fungi, benefits, costs, functional variability, genetic diversity, Glomus intraradices, intraspecific variation, plant growth, population, symbiosis.

Ecology Letters (2006) 9: 103-110

\section{INTRODUCTION}

Arbuscular mycorrhizal fungi (AMF) are abundant in soils of most terrestrial ecosystems where they form symbioses with roots of most plants (Smith \& Read 1997). Arbuscular mycorrhizal fungi (phylum Glomeromycota) are obligate biotrophs and supply plants with phosphorous in exchange for carbohydrates (Harrison 1997). The fungi are an ancient group of presumed asexual organisms and only c. 160 morphospecies are known (Redecker et al. 2000; Kuhn et al. 2001). Arbuscular mycorrhizal fungi grow clonally and are coenocytic, harbouring many nuclei in a common cytoplasm. The symbiosis with AMF can improve plant nutrient acquisition, enhance stress and pathogen tolerance and increase plant diversity (Grime et al. 1987; Newsham et al. 1995; Smith \& Read 1997).

Inoculation with different AMF species differentially alters the growth and coexistence of different plant species (Streitwolf-Engel et al. 1997; van der Heijden et al. 1998a, 2003) and increasing species richness of AMF increases plant diversity and productivity (van der Heijden et al. 1998b). However, these studies used single individuals as representatives of each AMF species where each culture was initiated from only one spore. These studies, therefore, did not address whether intraspecific variation could account for some of the observed differences among taxa. The question of inter- and intraspecific variation in AMF and its effects on plant growth has been partially addressed. Variation in plant growth was higher among plants inoculated with different species than those inoculated with different isolates of the same species (Hart \& Klironomos 2002). However, this does not mean that within isolate variation is not ecologically important. More recently considerable within AMF species variation has been observed. Large differences in plant growth and phosphorous uptake were reported within AMF species, thus, showing the potential ecological importance of within AMF species variation (Munkvold et al. 2004). The AMF isolates compared in the study by Munkvold et al. (2004) evolved in different environments and differed in their culturing histories. The isolates were probably also genetically different. Therefore, these large intraspecific differences could have been caused by environmental differences or genotypic differences or both. Different isolates of an AMF species from one geographic location have also been shown to cause variation in the amount of biologically fixed 
nitrogen in clover plants (Gamper et al. 2005). Recently, a population of $G$. intraradices was shown to be comprises of individuals that were genetically different from each other (Koch et al. 2004). These individuals were isolated from the same field and maintained for successive generations in exactly the same environment. These AMF isolates showed considerable differences in their amplified fragment length polymorphism (AFLP) banding patterns and their phenotypes, especially in extraradical hyphal density, a trait that is thought to play an important role in phosphorous acquisition. Variation in these traits likely has a genetic basis because culturing under identical conditions over several generations eliminated possible environmental effects.

The aim of the present study is to test whether genetic differences among individuals of an AMF population could significantly alter the growth of plants. In our experiments, we used isolates from a population of Glomus intraradices that had been maintained in the same environment (Koch et al. 2004) in order to reduce environmental effects. In a system where the AMF are grown with transformed carrot roots on an artificial medium, we tested whether genetically different isolates differentially affected root growth. In a second experiment, in the same culture system, we tested whether altering the environment would differentially alter the effect of a given isolate on root growth. In a greenhouse experiment, we tested whether the growth of two plant species, that had previously been shown to grow differently when forming the symbiosis with different AMF species (van der Heijden et al. 2003), was altered by the colonization of genetically different $G$. intraradices individuals that all come from one field (Koch et al. 2004). Because overall growth of AMF inoculated plants did not differ from that of non-mycorrhizal control plants in this first experiment, we performed a second greenhouse experiment, where a poorer soil mixture was used. To test whether differences in soil moisture altered growth and AMF-dependency of host plants, two different watering regimes were also included. In all experiments genetically different AMF isolates significantly altered host growth, and the effects ranged from detrimental to beneficial depending on the environmental conditions and the identity of the isolate. Our results show that ecologically relevant genetic and functional diversity exists within AMF populations revealing a further level of complexity of AMF biology and natural ecosystems.

\section{MATERIALS AND METHODS}

All fungal material used in our experiments belonged to the AMF species G. intraradices Schenck \& Smith. The species identification was based on spore morphology and internal transcribed spacer (ITS) sequences (Koch et al. 2004). The AMF single spore isolates originate from four different plots from one agricultural field in Tänikon, Switzerland (Anken et al. 2004; Koch et al. 2004). The agricultural field contained two replicate plots nested within two tillage treatments (plots A and B experienced no tillage and plots C and D experienced tillage). The distances among the plots varied from $29.2 \mathrm{~m}$ (distance from plot A to plot B) to $85.2 \mathrm{~m}$ (distance from plot B to plot C). Single G. intraradices spores were obtained from trap cultures that had been started from mixtures of soil samples from each of the four plots. These single spores were then placed individually onto germinating roots of Plantago lanceolata roots to start single spore cultures. From each of these cultures, a single healthy-looking spore was used to colonise transformed carrot roots in an axenic culture system. The isolates were subsequently kept under identical environmental conditions for two generations prior to experiment 1 and for at least seven generations before all other experiments to reduce possible maternal effects (Koch et al. 2004). The nomenclature of the fungal isolates used here is the same as in Koch et al. (2004); capitalized letters indicate the origin of the plot and the numbers indicate different single spore lines.

\section{Experiment 1: the effect of different isolates on host root growth}

The aim of experiment 1 was to test whether different isolates altered the growth of host roots. Sixteen AMF isolates (four isolates from each of the four plots) of G. intraradices were cultured in an axenic system with a clone of transformed carrot roots as described in Koch et al. (2004). Root length was measured 3, 6, 9, 12 and 15 weeks after inoculation of each plate by counting the number of root intersects with a rectangular grid $(2 \mathrm{~cm}$ interspace). The measures over time allowed the calculation of maximum root growth rate by determining the maximum slope of the least square fit of the logistic curve

$y=a(b+\exp (-c \times \text { week }))^{-1}$

for each of the 229 replicate plates of the experiment. For full details of experiment 1 see Koch et al. (2004).

\section{Experiment 2: the effect of decreased nutrient availability on host root growth}

The same model system as in experiment 1 was used to test how the growth of the host roots and four genetically different fungal isolates were altered when the availability of two key nutrients, sucrose and phosphate, was decreased. To do this, we selected four G. intraradices isolates, which were sampled from different plots and which were previously shown to differ in their AFLP fingerprint profiles (Koch et al. 2004). The four AMF isolates (A4, B3, C3 and D1) were cultivated in the standard $\mathrm{M}$ medium and in medium with reduced sucrose and phosphate concentra- 
tions. The concentration of either sucrose or potassium phosphate in the growth medium was reduced to $50 \%$ and $25 \%$ compared with the standard M medium respectively (Becard \& Fortin 1988). The reduced amount of potassium was compensated by adding the equivalent amount of $\mathrm{K}$ in the form of $\mathrm{KCl}$ to the medium. The five treatments are abbreviated as M (standard), S50, S25, for the reduced sucrose, and P50, P25 for the reduced phosphorous treatments respectively. After the end of experiment 1, the isolates and non-mycorrhizal (NM) roots were subcultured four times on $\mathrm{M}$ medium over a period of 1 year before being used for this experiment. For roots inoculated with each of the four isolates and the NM roots, 40 parental plates were randomized and used to inoculate 60 new plates, i.e. 12 new replicate plates of each of the five treatments. After 15 week of growth, root length of each plate was measured as in experiment 1 , and hyphal length and spore density were quantified as in Koch et al. (2004). Contaminated plates $(n=3)$ and plates with no AMF hyphal growth $(n=21)$ were removed from the statistical analyses, which reduced the total number of plates from 300 to 276 .

\section{Experiments 3: the effect of different isolates on total dry weight of two host species}

The aim of this greenhouse experiment was to test whether the growth of the two plant species Prunella vulgaris L. and Brachypodium pinnatum (L.) P.B. was altered when inoculated with different isolates of $G$. intraradices. To cover as much of the detected genetic diversity of this population as possible, we chose six isolates that were phenotypically and genetically the most different in a previous study (Koch et al. 2004). The seeds of the host plants were obtained from Fenaco (Winterthur, Switzerland). Seeds of the two host species were surface sterilized in $6 \%$ bleach for $10 \mathrm{~min}$, rinsed three times with sterile water and put on seed trays with moist vermiculite for germination in the greenhouse on 27 August 2002. A total of 140 pots $(10.5-\mathrm{cm}$ diameter $\times 8$-cm high) were filled with $450 \mathrm{~mL}$ of a $1: 1: 1$ (vol : vol : vol) mixture of a previously sieved (5-mm mesh width) high nutrient brown soil from an old field on the campus of the University of Lausanne (2684, 2.4 and $27 \mu \mathrm{mol} \mathrm{L}^{-1} \mathrm{~N}$, soluble $\mathrm{P}$ and $\mathrm{K}$ respectively), washed sand and pure quartz sand $(1 \mathrm{~mm})$. The soil mixture was steam autoclaved twice at $120{ }^{\circ} \mathrm{C} 2$ weeks before planting. On 8 September 2002, seedlings of similar size of either of the two host species were transferred to 70 pots that had been well watered. Two seedlings of the same species were transplanted per pot. Each seedling was inoculated with $0.2 \mathrm{~mL}$ spore suspension that contained 500 spores of one of the isolates A4, B1, B3, C2, C3 and D1, NM plants received $0.2 \mathrm{~mL}$ tap water. The spores used as inoculum were previously grown for 4 months with transformed carrot roots on split plates, and the cultures were kept under identical conditions for several years to eliminate maternal effects (Koch et al. 2004). After 16 days of growth the smaller of the two seedlings was removed. This resulted in 10 replicate pots for each isolate and NM treatment for each plant species. Each pot was watered every other day with $30 \mathrm{~mL}$ tap water. Day length was $16 \mathrm{~h}$, supplemented with artificial light when necessary. The temperatures ranged between 18 and $30^{\circ} \mathrm{C}$. The position of the individual pots was randomized weekly. The shoots of all plants were harvested 100 and 200 days after inoculation. This was done by cutting off all plant tissue $1 \mathrm{~cm}$ from the base of the shoot. This ensured re-growth by remaining meristems. At the final harvest, after 300310 days growth, the shoot of each host plant was separated from the roots and the roots were carefully washed. After recording the fresh weight of the roots, a small root sample was cut and stored in 50\% ethanol and the remaining fresh weight of the roots was recorded. At all harvests shoots and roots where separately dried at $80{ }^{\circ} \mathrm{C}$ for 2 days and then weighed. The stored root samples were stained with Trypan Blue as described in Munkvold et al. (2004) and inspected for AMF colonization. All plants survived to the end of the experiment and none of the NM plants showed any AMF colonization. All plants that received inoculum were clearly colonized by AMF.

\section{Experiment 4: the effect of different isolates on plant growth under two watering regimes}

The aim of this experiment was to test whether the same set of isolates used in experiment 3 also altered plant growth in conditions where there was a main positive effect of AMF inoculation on plant growth. In order to achieve this, we set-up two different watering regimes to test whether differences in soil moisture influenced AMF dependency on plant growth. The experimental procedures in experiment 4 were as in experiment 3 , except where indicated. The substrate mixture consisted of $3: 2$ (vol : vol) Terra Green (Oil Dry US Special, Maag Technic SA, Crissier, Switzerland) and pure quartz sand to which we added $4 \%$ (vol) of the same soil as in experiment 3. Single seedlings were inoculated and transplanted on 29 February 2004, using the same six AMF isolates, a NM treatment, and the same two 2-host species. For each host species and each AMF isolate and the NM treatment 16 replicate pots were used in the experiment. Day length was set at the natural day/night rhythm. Each pot was previously filled with $325 \mathrm{~g}$ of dried substrate and watered to water holding capacity (WHC) and later to $40-55 \%$ of WHC. In addition, eight replicate pots were established with each of the same two host plant species and each of the isolates A4, B3, and C2 and NM plants. These plants received an increased water regime where soil moisture was 
kept between $70 \%$ and $100 \%$ of WHC. All plants were watered with deionized water (Option 1; Lab Services AG, Wohlen, Switzerland). After 3 months, each pot was fertilized once with $10 \mathrm{~mL}$ of full-strength Hoagland solution containing no phosphorous and the $\mathrm{K}$ concentration was adjusted by adding $\mathrm{KCl}$. After 4 months, all shoots were harvested. The pots were subsequently stored in a cold room at $4{ }^{\circ} \mathrm{C}$. All the roots were washed and weighed within the following 3 weeks. One plant died during the experiment and in roots of four plants where inoculum was added no fungal colonization was observed. These five individuals were removed which left data from 283 plants for the analyses.

\section{Statistical analyses}

In experiment 1 , data were analysed using a nested ANOVA model with the main factors tillage treatment, plot (nested in treatment), isolate (nested in plot) and inoculation (plate nested in isolate), as in Koch et al. (2004). In the results, only significant main effects are given. In experiment 2 , data were analysed using a crossed two-way ANOVA with treatment and isolate as main fixed factors. The analyses were carried out separately for the different phosphorous and sucrose treatments and the root growth data was analysed both with and without NM roots. In experiments 1 and 2, Pearson's correlation coefficient was calculated for comparison of different growth variables. The data of experiments 3 and 4 were also analysed with a crossed two-way ANOVA with both host species and isolate as fixed factors. To test whether the overall growth of NM plants differed from AMF inoculated plants, all the isolates were pooled. In order to test for an AMF isolate by plant-species interaction, NM plants were omitted from the analysis. In experiment 4, data were analysed separately for the two water treatments. Variables were transformed, if necessary, to meet the requirements of the statistical tests (Zar 1984). All analyses were performed with the statistical programs JMP 5.0 (SAS Institute Inc., Cary, NC, USA) and R version 1.8.0 (R. Development Core Team, 2003, www.R-project.org).

\section{RESULTS}

\section{Experiment 1: the effect of different isolates on host root growth}

After 15-week growth, the length of transformed carrot roots differed significantly among isolates, with up to $15 \%$ difference in root length among different AMF isolate treatments (ANOva, $F_{12,46}=3.28, P<0.01$, Fig. 1 ). The maximal rate of root growth showed a similar pattern and was positively correlated with final root length $(r=0.76$, $P<0.0001$, data not shown). The maximal hyphal growth

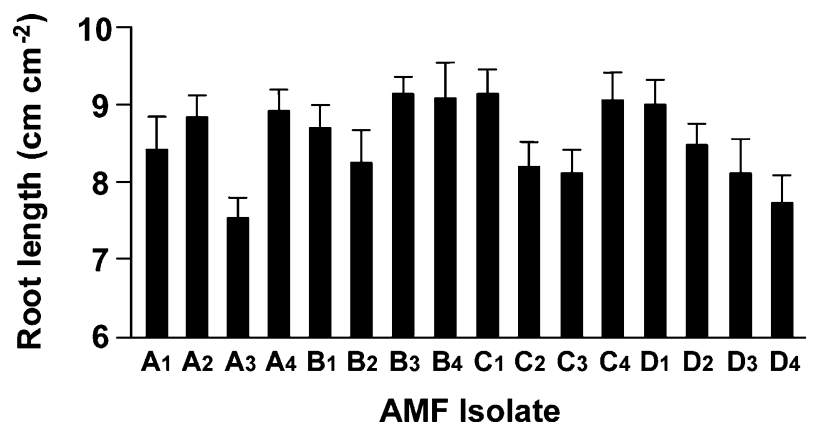

Figure 1 Mean final length of transformed $D$. carota roots after 15 weeks of growth in M medium and inoculated with 16 different single spore isolates of the AMF Glomus intraradices. Error bars represent $+1 \mathrm{SE}$.

rate was negatively correlated with both the maximal rate of growth of the roots $(r=-0.13, P<0.05)$ and the growth rate of the roots at the time of maximal hyphal growth $(r=$ $-0.19, P<0.01)$.

\section{Experiment 2: the effect of decreased nutrient availability on host root growth}

Growth of transformed carrot roots was significantly altered by both the reduced phosphorous concentration and the different AMF isolates $\left(F_{2,162}=5.23, P<0.01\right.$ and $F_{4,162}=9.07, P<0.0001$ respectively, Fig. 2 a). There was no significant $\mathrm{P}$-concentration by isolate interaction, indicating that root growth in the different phosphorous treatments responded in the same way with each of the different AMF isolates. The results were qualitatively the same when the NM roots were excluded from the analysis. Final hyphal length and spore density also differed among AMF isolates $\left(F_{3,129}=65.67, P<0.0001\right.$ and $F_{3,129}=$ 45.85, $P<0.0001$ respectively, Fig. $2 \mathrm{~b}, \mathrm{c})$ and among $\mathrm{P}$ concentrations $\left(F_{2,129}=5.64, P<0.01\right.$ and $F_{2,129}=7.68$, $P<0.001$ for the two variables respectively) with no significant interaction terms.

Growth of transformed carrot roots decreased considerably with the reduced sucrose concentrations (Fig. 2a, $F_{2,142}=247.35, \quad P<0.0001 \quad$ and $\quad F_{2,109}=209.07$, $P<0.0001$, with and without NM roots respectively). Root growth also differed among isolates $\left(F_{4,142}=7.00\right.$, $P<0.0001$ and $F_{3,109}=8.87, P<0.0001$, with and without NM roots respectively, Fig. 2a). The growth of NM roots was lower than inoculated roots in $\mathrm{M}$ medium. However, the growth of NM roots was higher than some AMF inoculated roots in medium with lowered sucrose concentration, which resulted in a significant sucrose concentration by AMF isolate interaction $\left(F_{8,142}=2.79\right.$, $P<0.01$, Fig. 2a). The interaction term was not significant when the NM roots were omitted from the analysis. Hyphal 
(a)

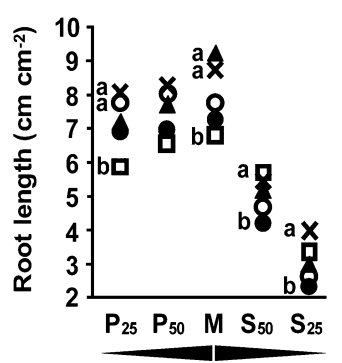

(b)

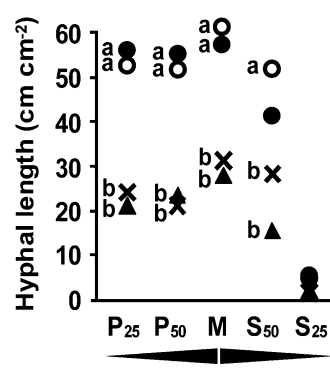

(c)

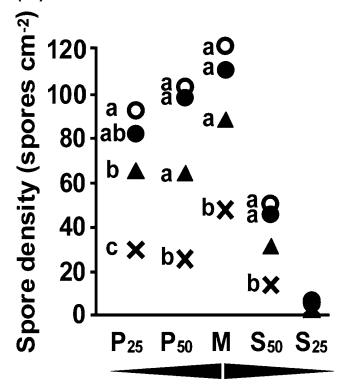

\section{Medium composition}

Figure 2 (a) Mean final length of transformed D. carota roots, (b) mean hyphal length and (c) mean spore density of four Glomus intraradices isolates. Transformed $D$. carota roots were each colonized with one of four genetically different arbuscular mycorrhizal fungi (AMF) isolates of G. intraradices (solid circle A4, black triangle B3, open circle C3, cross D1) or non-mycorrhizal (open squares). The roots were grown on five different media; standard M medium (M), M medium with phosphate reduced to $50 \%$ (P50), phosphate reduced to $25 \%$ (P25), sucrose reduced to $50 \%(\mathrm{~S} 50)$ and sucrose reduced to $25 \%$ (S25). Although data from phosphorous and sucrose treatments are graphed together, the black-shaded triangles below each graph indicate that ANOvAs were performed separately for the different phosphate and sucrose concentrations and both analyses included the standard M medium. Different letters left of symbols of the same medium composition indicate a significant difference $(P<0.05)$ according to the Tukey-Kramer honest significant test $(\mathrm{HSD}, P<0.05)$.

length and spore density differed among isolates $\left(F_{3,109}=\right.$ 17.44, $P<0.0001$ and $F_{3,109}=11.08, P<0.0001$ respectively, Fig. $2 b, c)$. Hyphal length and spore density also differed greatly in the different sucrose concentrations $\left(F_{2,109}=207.41, P<0.0001\right.$ and $F_{2,109}=230.73, P<$ 0.0001 respectively) with growth being much lower at low sucrose concentrations compared with the $\mathrm{M}$ medium. There was no significant AMF isolate by sucrose concentration interaction for either variable.

When all isolates were pooled, hyphal length and spore density were positively correlated in each of the five treatments of different nutrient availability $(P<0.0001$, for all five treatments). However, there were significant negative correlations between hyphal and root length on $\mathrm{M}$ medium $(r=-0.37, P<0.05)$ and between spore density and root length on M medium $(r=-0.34, P<0.05)$ and in the S50 treatment $(r=-0.32, P<0.05)$.

\section{Experiment 3: the effect of different isolates on total dry weight of two host species}

After 10 months of growth in the green house, there was no significant effect of mycorrhizal inoculation on the total dry weight of either plant species when comparing AMF inoculated with NM plants (Fig. 3). However, inoculation with different $\mathrm{AMF}$ isolates significantly altered the total dry weight of the plants $\left(F_{5,108}=4.50, P<0.0001\right.$, Fig. 3$)$. The total dry weight of $B$. pinnatum plants was greater than that of $P$. vulgaris $\left(F_{1,108}=44.82, P<0.0001\right)$ and there was no significant isolate by host interaction. All plants that received AMF inoculum were clearly colonized and no AMF

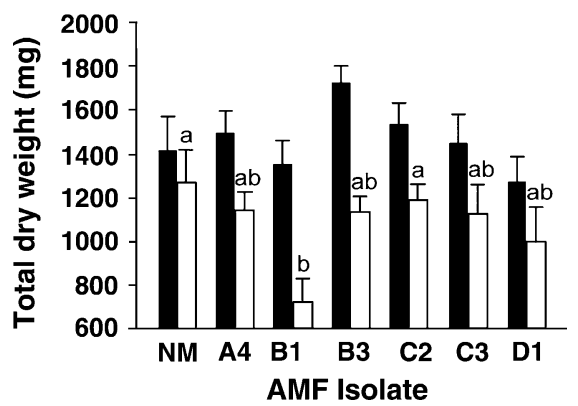

Figure 3 Mean total dry weight of plants that were inoculated with six genetically different Glomus intraradices isolates (A4, B1, B3, C2, C3 and D1) or left uninoculated [non-mycorrhizal (NM)] after a growth period of 10 months. Two host species, Brachypodium pinnatum (black shading) and Prunella vulgaris (unshaded), were used in the experiment. Error bars represent $+1 \mathrm{SE}$ and different letters above bars of the same plant species indicate a significant difference $(P<0.05)$ according to the Tukey-Kramer honest significant test (HSD) test.

colonization was observed in any of the NM plants. The $P$. vulgaris plants inoculated with isolate $\mathrm{B} 1$ tended to be smaller than plants colonized with other $G$. intraradices isolates as well as non-mycorrhizal plants at all three harvests. In contrast, at all three harvests $B$. pinnatum plants colonized with isolate B3 were larger than plants colonized with the other isolates and NM plants (data not shown). The total dry weight of the roots was strongly correlated with the total dry weight of the shoots for both host species $(r=0.84$, $P<0.0001$ and $r=0.82, P<0.0001$ for B. pinnatum and P. vulgaris respectively). 


\section{Experiment 4: the effect of different isolates on plant growth under two watering regimes}

At the end of experiment 4, the roots of all plants that had received AMF inoculum were colonized with AMF and none of the NM plants were colonized with AMF. In contrast to experiment 3, AMF colonized plants grew significantly larger than NM plants after a growth period of 4 months under dry conditions $\left(F_{1,215}=146.42\right.$, $P<0.0001$, Fig. 4a). The AMF growth enhancement was
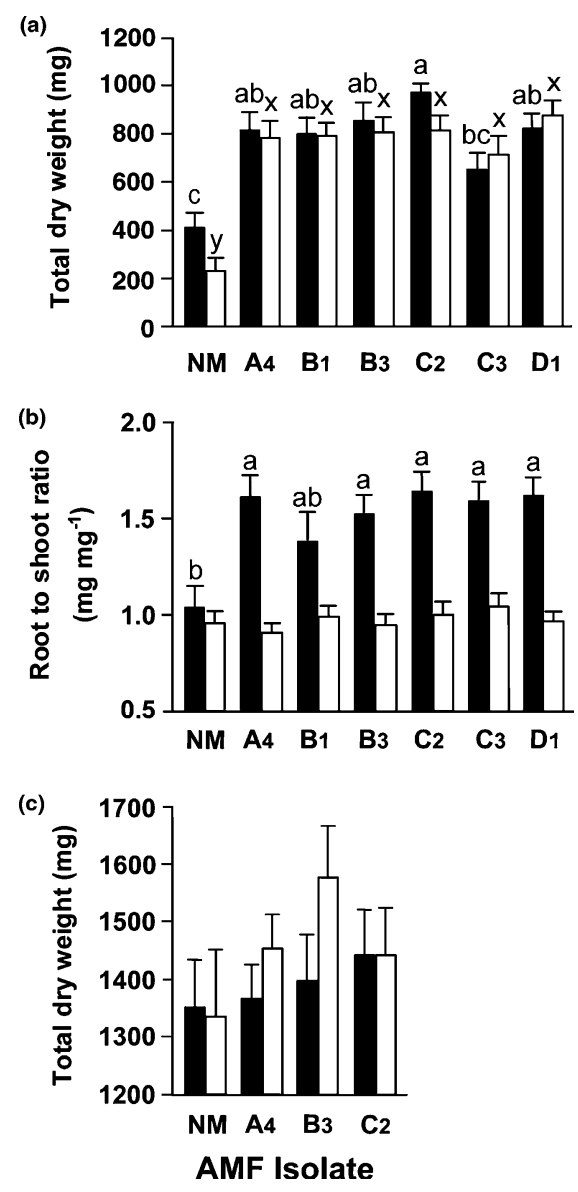

Figure 4 (a) Mean total dry weight and (b) root to shoot ratio of plants that were inoculated with six genetically different Glomus intraradices isolates (A4, B1, B3, C2, C3 and D1) or left uninoculated [non-mycorrhizal (NM)]. Values were at the final harvest 4 months after inoculation and under dry growth conditions (40-55\% of soil water holding capacity, $n=219$ ). (c) Mean total dry weight of NM plants and plants inoculated with three arbuscular mycorrhizal fungi (AMF) isolates (A4, B3 and C2) and 4 months after inoculation, under wet growth conditions $(70-100 \%$ of WHC, $n=64)$. Two host species, Brachypodium pinnatum (black shading) and Prunella vulgaris (unshaded), were used in the experiment. Error bars represent $+1 \mathrm{SE}$ and different letters above bars of the same plant species indicate a significant difference $(P<0.05)$ according to the Tukey-Kramer honest significant test (HSD) test. greater for P. vulgaris than B. pinnatum. Plants colonized with isolate C2 were larger than plants colonized with isolate C3 and overall the AMF isolates significantly altered final total plant dry weight $\left(F_{5,175}=3.01, \quad P<0.05\right)$ with no significant host effect and isolate by host interaction. Mycorrhizal B. pinnatum plants had a significantly higher root to shoot ratio than $P$. vulgaris $\left(F_{1,175}=185.98\right.$, $P<0.0001$, Fig. 4b) and there was no significant isolate effect or isolate by host interaction. The absence of a significant isolate by host interaction indicates that the growth response of the two plant species did not differ according to which isolate they had been inoculated with. Under wetter growth conditions the plants grew larger than under dry growth conditions (Fig. 4a, c). Plants inoculated with AMF were colonized and tended to be larger than the $\mathrm{NM}$ plants, which were not colonized with $\mathrm{AMF}\left(F_{1,60}=\right.$ 2.80, $P<0.1$, Fig. 4c), but there were no significant isolate, host or interaction effects.

\section{DISCUSSION}

Our results (experiments 1-4) show that genetically different individuals of $G$. intraradices from a population originating from one field alter plant growth. Using an artificial study system where AMF isolates grow with transformed carrot roots we found, in both experiments 1 and 2, that genetically different AMF isolates significantly influenced root length of transformed Daucus carota. The results from experiment 2 additionally show that altering the environment can result in AMF colonization that is costly for the plant. We also conducted two experiments in the greenhouse where, in both cases, genetically different AMF isolates significantly altered plant growth. In experiment 3, although there was no overall mycorrhizal benefit of the plants (comparison of NM with AMF inoculated plants) genetically different AMF isolates resulted in differential plant growth. Costs of AMF colonization were also seen for $P$. vulgaris plants colonized with one isolate. In experiment 4, where all AMF isolates enhanced plant growth compared with NM plants, different isolates also altered plant growth.

In this study, we have shown that significant functional variability exists in an AMF population. The study by Munkvold et al. (2004) showed large differences in plant phosphorous uptake, which were caused by isolates from different geographic origins. Compared with our study, the AMF isolate effects observed by Munkvold et al. (2004) appear larger and this points to the fact that the origin of an isolate also plays an important role. It is also possible that the genetic differences among AMF individuals used by Munkvold et al. (2004) from different regions were larger than the genetic differences among individuals within the population used in our study. Koch et al. (2004) have already shown 
spatial genetic structure in an AMF population. Combined with our results here, and the findings of Munkvold et al. (2004), this suggests that both genetic and functional diversity in AMF species is spatially structured both within and among populations. A sampling scheme of AMF populations from a local to a regional or even global scale would be required to address the question of how functional and genetic diversity is related to different spatial scales. Only by sampling several individuals, of several species and genera from one community would it be possible to address the question at what taxonomic level most functional AMF diversity is explained. To our knowledge, no such experiment has yet been performed, but the results from Hart \& Klironomos (2002) indicate that variation between AMF genera is higher than within the genus Glomus.

In experiment 2, reduction of two key nutrients (phosphate and sucrose) in the growth medium decreased root and fungal growth, but the effects were much stronger when sugar was limiting. Under such limiting conditions of reduced sucrose availability AMF colonized roots had a reduced root length compared with NM roots. This indicates that there are costs of the symbiosis for the plant roots under these environmental conditions. This is also supported by negative correlations between root and fungal growth in both experiments 1 and 2 . In experiment 2 , however, this was only the case on the $\mathrm{M}$ medium (highest nutrient availability) and in the S50 treatment (50\% reduced sucrose availability). This indicates that nutrient availability influences costs and benefits as well as potential conflicts between the growth potential of host and fungal symbionts. These findings also suggest that the host plant does not strictly control the symbiosis because AMF colonization continued under decreased resource availability despite increased costs to the host. However, overall fungal and root growth were reduced under decreased resource availability, but the growth reduction on fungal growth appear larger than that of roots in the treatment of lowest sucrose availability. This indicates that there are thresholds of nutrient availability allowing sustainable growth of both symbionts. This culture system is, therefore, ideal to further investigate the conditions and consequences of $\mathrm{AMF}$ and host root co-existence in controlled experiments. The fact that we detected no significant AMF isolate by environment effect in experiment 2 indicates that the genetically different isolates did not differentially effect root growth in these treatments of reduced sucrose and phosphate availability. However, it is still possible that other AMF isolates from this population, that were not used in this study, could differentially modify their growth in response different environments.

There was also no significant host species by AMF isolate interaction in neither experiment 3 nor experiment 4 indicating that these isolates did not have specific host species affinities that resulted in differential plant growth. Evidence for selection for similar phenotypes in this AMF population (Koch et al. 2004) could explain similar function and thus the relatively consistent performance of the isolates.

Our results are also interesting in the light of the fact that all AMF isolates used in this study come from an agricultural field. There is evidence showing that AMF from nutrientrich environments such as fertilized soils tend to be less efficient symbionts (Johnson 1993). While, overall there was no AMF inoculation effect in experiment 3, one AMF isolate (B1) significantly reduced plant growth of $P$. vulgaris, but had no such drastic negative effect on the second host species B. pinnatum. In experiment 4, where the soil mixture was poorer in nutrients and the growth conditions drier, all G. intraradices isolates used enhanced plant growth showing that all these individuals have maintained functions, which can be beneficial to their hosts. It will be interesting to further investigate whether and how environmental differences or different host species influence, interact with or possibly maintain the genetic diversity present in AMF populations.

In general, however, the AMF symbiosis is thought to be mutualistic as it can improve plant growth and nutrient acquisition, protect hosts from pathogens, alter hostherbivore interactions, and increase plant drought tolerance (Newsham et al. 1995; Smith \& Read 1997; Auge 2001; Gange et al. 2003; Garmendia et al. 2005; Kula et al. 2005). Nevertheless, plants that form the AMF symbiosis often have no growth benefit or even a reduced growth Johnson et al. 1997; Jifon et al. 2002; Klironomos 2003; Kytöviita et al. 2003; Brundrett 2004). The frequent observation of commensal or parasitic AMF-plant interaction could also be an insurance policy for plants, as in an unpredictable and changing environment short-term costs of plants could be outweighed by long-term net benefits. Our results show that genetic variability in an AMF population cause a range of different outcomes of plant growth, which also depend on environmental conditions. We conclude that intraspecific variability and genetic diversity in AMF populations cause variation in plant growth, which could also be ecologically relevant on the ecosystem level and be important for the development of potent AMF inocula for successful restoration of plant communities.

\section{ACKNOWLEDGEMENTS}

We thank Nicoletta Rinaudo, Gerrit Kuhn and Martine Ehinger for their help in the greenhouse and the Swiss National Science foundation (project 3100AO-105790/1) to which support is gratefully acknowledged. We appreciate the constructive comments of three referees that helped to improve this manuscript. 


\section{REFERENCES}

Anken, T., Weisskopf, P., Zihlmann, U., Forrer, H., Jansa, J. \& Perhacova, K. (2004). Long-term tillage system effects under moist cool conditions in Switzerland. Soil Tillage Res., 78, 171-183.

Auge, R.M. (2001). Water relations, drought and vesiculararbuscular mycorrhizal symbiosis. Mycorrbiza, 11, 3-42.

Becard, G. \& Fortin, J.A. (1988). Early events of vesicular arbuscular mycorrhiza formation on Ri T-DNA transformed roots. New Phytol., 108, 211-218.

Brundrett, M. (2004). Diversity and classification of mycorrhizal associations. Biol. Rev., 79, 473-495.

Gamper, H., Hartwig, U.A. \& Leuchtmann, A. (2005). Mycorrhizas improve nitrogen nutrition of Trifolium repens after $8 \mathrm{yr}$ of selection under elevated atmospheric $\mathrm{CO}_{2}$ partial pressure. New Phytol., 167, 531-542.

Gange, A.C., Brown, V.K. \& Aplin, D.M. (2003). Multitrophic links between arbuscular mycorrhizal fungi and insect parasitoids. Ecol. Lett., 6, 1051-1055.

Garmendia, I., Goicoechea, N. \& Aguirreolea, J. (2005). Moderate drought influences the effect of arbuscular mycorrhizal fungi as biocontrol agents against Verticillium-induced wilt in pepper. Mycorrbiza, 15, 345-356.

Grime, J.P., Mackey, J.M.L., Hillier, S.H. \& Read, D.J. (1987). Floristic diversity in a model system using experimental microcosms. Nature, 328, 420-422.

Harrison, M.J. (1997). The arbuscular mycorrhizal symbiosis: an underground association. Trends Plant Sci., 2, 54-60.

Hart, M.M. \& Klironomos, J.N. (2002). Diversity of arbuscular mycorrhizal fungi and ecosystem functioning. In: Mycorrbizal Ecology (eds van der Heijden, M.G.A. \& Sanders, I.R.). Stud. Ecol., 157. Springer-Verlag, Heidelberg, pp. 225-242.

van der Heijden, M.G.A., Boller, T., Wiemken, A. \& Sanders, I.R. (1998a). Different arbuscular mycorrhizal fungal species are potential determinants of plant community structure. Ecology, 79, 2082-2091.

van der Heijden, M.G.A., Klironomos, J.N., Ursic, M., Moutoglis, P., Streitwolf-Engel, R., Boller, T. et al. (1998b). Mycorrhizal fungal diversity determines plant biodiversity, ecosystem variability and productivity. Nature, 396, 69-72.

van der Heijden, M.G.A., Wiemken, A. \& Sanders, I.R. (2003). Different arbuscular mycorrhizal fungi alter coexistence and resource distribution between co-occurring plants. New Phytol., 157, 569-578.

Jifon, J.L., Graham, J.H., Drouillard, D.L. \& Syvertsen, J.P. (2002). Growth depression of mycorrhizal citrus seedlings grown at high phosphorus supply is mitigated by elevated $\mathrm{CO}_{2}$. New Phytol., 153, 133-142.

Johnson, N.C. (1993). Can fertilization of soil select less mutualistic mycorrhizae? Ecol. Appl., 3, 749-757.

Johnson, N.C., Graham, J.H. \& Smith, F.A. (1997). Functioning of mycorrhizal associations along the mutualism-parasitism continuum. New Phytol., 135, 575-586.

Klironomos, J.N. (2003). Variation in plant response to native and exotic arbuscular mycorrhizal fungi. Ecology, 84, 2292-2301.

Koch, A.M., Kuhn, G., Fontanillas, P., Fumagalli, L., Goudet, I. \& Sanders, I.R. (2004). High genetic variability and low local diversity in a population of arbuscular mycorrhizal fungi. Proc. Natl Acad. Sci. USA, 101, 2369-2374.

Kuhn, G., Hijri, M. \& Sanders, I.R. (2001). Evidence for the evolution of multiple genomes in arbuscular mycorrhizal fungi. Nature, 414, 745-748.

Kula, A.A.R., Hartnett, D.C. \& Wilson, G.W.T. (2005). Effects of mycorrhizal symbiosis on tallgrass prairie plant-herbivore interactions. Ecol. Lett., 8, 61-69.

Kytöviita, M.M., Vestberg, M. \& Tuomi, J. (2003). A test of mutual aid in common mycorrhizal networks: established vegetation negates benefit in seedlings. Ecology, 84, 898-906.

Munkvold, L., Kjoller, R., Vestberg, M., Rosendahl, S. \& Jakobsen, I. (2004). High functional diversity within species of arbuscular mycorrhizal fungi. New Phytol., 164, 357-364.

Newsham, K.K., Fitter, A.H. \& Watkinson, A.R. (1995). Arbuscular mycorrhiza protect an annual grass from root pathogenic fungi in the field. J. Ecol., 83, 991-1000.

Redecker, D., Kodner, R. \& Graham, L.E. (2000). Glomalean fungi from the ordovician. Science, 289, 1920-1921.

Smith, S.E. \& Read, D.J. (1997). Mycorrbizal Symbiosis. Academic Press, London, UK.

Streitwolf-Engel, R., Boller, T., Wiemken, A. \& Sanders, I.R. (1997). Clonal growth traits of two Prunella species are determined by co-occurring arbuscular mycorrhizal fungi from a calcareous grassland. J. Ecol., 85, 181-191.

Zar, J.H. (1984). Biostatistical Analysis, 2nd edn. Prentice-Hall, Englewood Cliffs, NJ, USA.

Editor, Rebecca Irwin

Manuscript received 3 August 2005

First decision made 14 September 2005

Manuscript accepted 3 October 2005 